\title{
Möglichkeiten der Haftungsbegrenzung in Allgemeinen Vertragsbedingungen zur Kaufuntersuchung
}

\author{
Dr. Dietrich Plewa \\ Kanzlei Dr. Plewa \& Doppler, Germersheim
}

Das Vertragsrecht wird vom Grundsatz der Vertragsfreiheit beherrscht. Der Begriff umfasst die Freiheit des Einzelnen, selbst darüber zu entscheiden, ob und mit welchem Inhalt er einen Vertrag abschließen will (Rechtslexikon). Die Vertragsfreiheit besteht im Wesentlichen aus zwei Elementen, nämlich der Abschluss- und der Gestaltungsfreiheit. Erstere umfasst die Entscheidung, ob überhaupt ein Vertrag abgeschlossen werden soll. Das Recht, von einem Vertragsabschluss abzusehen, steht grundsätzlich auch dem Tierarzt zu, speziell bei der Kaufuntersuchung, bei der es nicht um eine therapeutische, sondern um eine gutachterliche Tätigkeit des Tierarztes geht. Einschränkungen in der Abschlussfreiheit gibt es nur in Ausnahmefällen, die jedoch im Rahmen der Kaufuntersuchung nicht zum Tragen kommen. Weiteres Element der Vertragsfreiheit ist die inhaltliche Gestaltungsfreiheit. Sie umfasst das Recht der Vertragsparteien, einen Vertrag nach den beiderseitigen Interessenlagen auszugestalten, beispielsweise Regelungen zu den durch den Vertragsabschluss begründeten Hauptpflichten zu treffen, aber etwa auch zu Fragen der Fälligkeit der zu erbringenden Leistungen oder zur Haftung des Vertragspartners, der verschuldet oder unverschuldet die ihm obliegende leistung nicht oder nicht vollständig erbringt. Die Vertragsfreiheit ist die Haupterscheinungsform der Privatautonomie und als solche verfassungsrechtlich geschütz† (Palandt 2007).

Im Rahmen der Gestaltungsfreiheit können zwischen Tierarz† und Auftraggeber individuelle Vereinbarungen auch zur Haftungsbegrenzung getroffen werden. Sie können sich dabei sowohl auf die Höchstsumme zu leistenden Schadensersatzes als auch auf die Länge der Veriährungsfrist für vertragliche Schadensersatzansprüche erstrecken. Diese Möglichkeit bedarf allerdings keiner Vertiefung, weil in der Praxis individuell ausgehandelte Verträge speziell zu einem sehr häufig vorkommenden Vertragstypus wie dem der Kaufuntersuchung (Plewa 2002) eine seltene Ausnahme darstellen.

Wegen der praktischen Relevanz und Häufigkeit des Kaufuntersuchungsvertrages bedient sich der Tierarzł regelmäßig vorformulierter Vertragsbedingungen, die rechtlich als Allgemeine Geschäftsbedingungen (AGB) eingestuft werden (so: Pferdeheilkunde - Vertragsformular). Die inhaltliche Gestaltungsfreiheit unterliegt geradezu selbstverständlichen Schranken dahingehend, dass ein Rechtsgeschäft nicht gegen ein gesetzliches Verbot oder die "guten Sitten" verstoßen darf ( $\S \S$ 134, 138 BGB). Dieser Grundsatz kann zwar im Pferdekauf eine Rolle spielen, weil im Einzelfall ein sittenwidrig überhöhter Kaufpreis zur Nichtigkeit eines Kaufvertrages führen kann (BGH 2002), der Maßstab ist jedoch für die Beurteilung der Vertragsbedingungen des Formularvertrages für die Kaufuntersuchung eines Pferdes nicht entscheidend, sieht man davon $a b$, dass schon aus standesrechtlichen Gründen ein Aus- schluss für vorsätzliches oder grob fahrlässiges Verhalten des Tierarztes wegen Verstoßes gegen $\S 138$ BGB unwirksam sein könnte (Eikmeier et.al. 1990). Einer Überprüfung am Maßstab der $\S \S 134,138$ BGB bedarf es jedoch schon deswegen nicht, weil ein spezieller Prüfungsmaßstab für AGB in den $\S \S 307 \mathrm{ff}$. BGB vorgegeben ist. Im Rahmen der Schuldrechtsmodernisierung, die uns auch das neue Kaufrecht beschert hat, wurde das vormalige AGB-Gesetz in das BGB integriert. Voraussetzung dafür, dass von Allgemeinen Geschäftsbedingungen die Rede sein kann, ist die Verwendung vorformulierter Vertragsbedingungen in einer Vielzahl von Verträgen, § 305 BGB. Sie kommen allerdings nur dann zur Geltung, wenn sie in den Ve rtrag einbezogen werden. Das wiederum erfordert, dass die andere Vertragspartei ausdrücklich auf den Inhalt der AGB hingewiesen worden und mit ihrer Geltung einverstanden ist, $\S 305$ Abs. 2 BGB (Palandt 2007, Rz 25).

In aller Regel wird also der Tierarzt darauf zu achten haben, dass sein Auftraggeber das Vertragsformular zumindest zur Kenntnis nimmt. Der Nachweis dieser Information und des Einverständnisses wird am leichtesten dann gelingen, wenn der Auftraggeber das Vertragsformular einschließlich der AGB unterzeichnet hat.

An Stelle oder auch neben vorformulierten Vertragsbedingungen können individuelle Absprachen getroffen werden, die grundsätzlich Vorrang vor AGB genießen. Voraussetzung ist jedoch, dass die Vereinbarung tatsächlich und nachweisbar ausgehandelt wurde. Sie kann sich dann aus hand- oder maschinenschriftlichen Einfügungen oder einem Bestätigungsschreiben ergeben (BGH 1987, 1995). An den Nachweis des Aushandelns einer Individualabrede werden allerdings von der Rechtsprechung strenge Anforderungen gestellt, weshalb es sich empfiehlt, derartige Vereinbarungen handschriftlich festzuhalten. Steht dann allerdings eine Individualabrede inhaltlich im Widerspruch zu einer Bestimmung des Formularvertrages, so ist letztere unwirksam (Palandt 2007, Rz 3).

Der eine Kaufuntersuchung durchführende Tierarzt wird in der Regel ein Interesse daran haben, sein Haftungsrisiko zu begrenzen, zumal unverändert ein völlig unangemessenes Verhältnis zwischen der Höhe des tierärztlichen Honorars und dem Umfang der Schadensersatzpflicht bei fehlerhafter Kaufuntersuchung besteht (Plewa 1987). Eine derartige Eingrenzung des Risikos kann sowohl in der Verkürzung der Veriährungsfrist für Schadensersatzansprüche als auch in der Begrenzung der Haftungshöchstsumme bzw. der Beschränkung der Haftung auf grobes Verschulden liegen. Den Gestaltungsmöglichkeiten im Rahmen formularmäßiger Bedingungen sind allerdings enge Grenzen gesetzt. Das hat ein aktuelles Urteil des BGH im Besonderen deutlich aufgezeigt (BGH 2006). Der BGH hatte 
sich mit den Vertragsbedingungen einer Fohlenauktion zu befassen. In den AGB der Auktion war die gesetzliche Veriährungsfrist für Ansprüche des Käufers von zwei Jahren auf ein Jahr verkürzt worden. Der BGH hielt diese formularmäßige Verkürzung für unwirksam. Interessant ist insoweit die Begründung. Der BGH hat nämlich nicht etwa erklärt, dass generell in AGB eine Verkürzung der Veriährungsfrist unzulässig sei. Im Gegenteil: Aus seinem Urteil lässt sich durchaus entnehmen, dass unter Einhaltung bestimmter Voraussetzungen die Veriährungsfrist formularmäßig verkürzł werden kann. Für die Ansprüche des Käufers wegen einer fehlerhaften Kaufuntersuchung gilt die allgemeine Veriährungsfrist von drei Jahren, § 634 a Abs. 1 Z. 3 BGB. Seit Inkrafttreten des Schuldrechtsmodernisierungsgesetzes kann entgegen der früheren Gesetzeslage diese Verjährungsfrist nicht auf weniger als ein Jahr verkürzt werden, § 309 Nr. 8 lit. b) ff) BGB (Plewa 2005). Damit nicht genug: Von dem Verbot, zu Lasten des Vertragspartners die Verährungsfrist zu verkürzen, ist auch das Verbot umfasst, den Veriährungsbeginn abweichend vom Gesetz zu regeln. Dies wiederum ergibt sich aus $\S$ 199. Danach beginnt die regelmäßige Veriährungsfrist mit dem Schluss des Jahres, in welchem der Anspruch entstanden ist und der Gläubiger von den, den Anspruch begründenden Umständen und der Person des Schuldners Kenntnis erlangt oder ohne grobe Fahrlässigkeit erlangen musste. Diese Regelung ist zwingend mit der Folge, dass in jedem Fall der Beginn der Veriährungsfrist kenntnisabhängig ist. Unabhängig von der Kenntnis kann die Veriährungsfrist allenfalls auf fünf Jahre verkürzt werden (Plewa 2005).

Damit ist allerdings den vom BGH ausdrücklich hervorgehobenen Anforderungen immer noch nicht genüge getan. Der $\mathrm{BGH}$ sieht nämlich in der Verkürzung der gesetzlichen Veriährungsfrist eine Haftungsbeschränkung (BGH 2006; Palandt § 309). Insoweit ist die Verkürzung der Veriährungsfrist beispielsweise der Begrenzung der Haftung auf die Fälle groben Verschuldens gleichzustellen. Hierzu besagt $\S 309 \mathrm{Nr}$. 7 lit. a und b BGB, dass in Allgemeinen Geschäftsbedingungen unwirksam solche Klauseln sind, mit denen die Haftung für die Verletzung von Leben, Körper, Gesundheit und für grobes Verschulden, also eine vorsätzliche oder grob fahrlässige Pflichtverletzung, ausgeschlossen wird. Auch wenn kaum vorstellbar ist, dass durch eine fehlerhafte Kaufuntersuchung ein Personenschaden verursacht werden kann (ein solcher Fall ist, soweit ersichtlich, bisher nicht aufgetreten, jedenfalls nicht gerichtlich entschieden worden), so müssen AGB dennoch der gesetzlichen Vorgabe Rechnung tragen. In der erwähnten Entscheidung des $B G H$ heißt es nämlich sinngemäß:

Eine Klausel in Allgemeinen Geschäftsbedingungen, mit denen die gesetzliche Verjährungsfrist abgekürzt wird, ist wegen Verstoßes gegen die Klauselverbote des $\S 309$ Nr. 7 Buchst. a und b BGB insgesamt unwirksam, wenn die in diesen Klauselverboten bezeichneten Schadensersatzansprüche nicht von der Abkürzung der Veriährungsfrist ausgenommen werden (BGH 2006). Mit anderen Worten: In den AGB zur Kaufuntersuchung müsste, um deren Wirksamkeit insgesamt sicherzustellen, von der Haftungsbegrenzung ausdrücklich ausgenommen werden die Haftung des Tierarztes für Personenschäden, außerdem für Schadensersatzansprüche des Auftraggebers wegen einer grob fahrlässig oder gar vorsätzlich begangenen Pflichtverletzung des Tierarztes oder eines für ihn tätigen Erfüllungsgehilfen. Mi† dieser Maßgabe allerdings wäre dann die Haftungsbeschränkung durchaus möglich (Graf von Westphalen 2005).
Auch bei Beachtung der erwähnten Voraussetzungen bleibt immer noch zweifelhaft, ob die Haftung für die so genannten Hauptpflichten, auch Kardinalpflichten genannt, beschränkt werden kann. Zu den Kardinalpflichten gehört sicherlich die Feststellung der erheblichen Befunde. Wenn allerdings insoweit eine Haftungsbegrenzung generell unzulässig wäre, würde jeder Versuch der Tierärzteschaft, das Haftungsrisiko im Rahmen der Kaufuntersuchung durch AGB einzuschränken, ins Leere gehen (Kappus 2006). Das Ergebnis erschiene keinesfalls interessengerecht, weshalb die Rechtsprechung bislang Haftungsbegrenzungen in den AGB zur Kaufuntersuchung auch durchaus toleriert hat (LG Lübeck 2002, LG Hannover 1992, LG Fulda 1999, OLG München 1994, LG Kaiserslautern 2005). Soweit der Tierarzt in den Vertragsbedingungen wirksam seine Haftung eingeschränkt hat, gilt diese Haftungsbegrenzung auch gegenüber einem in den Schutzbereich einzubeziehenden Dritten. Das bedeutet beispielsweise: Hat der Käufer den Auftrag zur Kaufuntersuchung erteilt, so ist auch der Käufer in den Schutzbereich des Werkvertrages einzubeziehen. Er kann also Schadensersatzansprüche gegenüber dem Tierarzt aus der Verletzung einer vertraglichen Pflicht herleiten. Allerdings gelten dann auch ihm gegenüber die Haftungsbegrenzungen, die - wirksam - zwischen dem Tierarzt und dem Verkäufer als Auftraggeber vereinbart wurden (OLG München 1994). Im Gegensatz zur Rechtsprechung und Literatur vertritt Graf von Westphalen (2004) eine abweichende Meinung.

\section{Literatur}

BGH (2002): Urteil vom 18.12.2002, Az. VIII ZR 123/02, Deutsches Rechtslexikon, 2. Auflage, Band 3

BGH (2006): Urteil vom 15.11.2006, Az. VIII ZR 3/03BGH Neve Juristische Wochenschrift (NJW) 87, 2011; NJW-Rechtsprechungsreport 95, 179

Deutsches Rechtslexikon (1992): Stichwort: Vertragsfreiheit. 2. Auflage, Beck-Verlag, Band 3

Eikmeier H., Fellmer E. und Moegle H. (1990): Lehrbuch der gerichtlichen Tierheilkunde, Paul Parey Verlag, 1. Auflage72

Graf von Westphalen E. (2004): www.hufgeflüster.de

Graf von Westphalen E. (2005): Tierärztl. Praxis 2005, 197-199

Kappus A. (2006): BGH "succurit ignorante" - Transparenz des "Kardinalpflichten"-Begriffs im Unternehmerverkehr. NJW, 15

LG Fulda (1991): Urteil vom 28.05.1991, Az. 2 O 486/98

LG Hannover (1992): Urteil vom 15.07.1992 Az. 18 O 52/92

LG Kaiserslautern (2005): Urteil vom 09.11.2005, Az. 4 O 1084/03

LG Lübeck (2002): Urteil vom 26.04.2002, Az. 5 ○ 251/01

OLG München (1994): Urteil vom 06.12.1994, Az. 25 U 4042/94

OLG München (2004): www.hufgeflüster.de

Palandt O. (2007): Bürgerliches Gesetzbuch, 66. Auflage, Rz 25 zu $\S 305$ BGB, Rz 3 zu § 305 b BGB, Rz 78 zu § 309 BGB, Rz. 7 zu Einführung vor $\S 145 \mathrm{BGB}$

Plewa D. (1987): Vertrag über die Untersuchung eines Pferdes, Pferdeheilkunde 3, 297-298

Plewa D. (2002): Die Kaufuntersuchung des Pferdes aus rechtlicher Sicht. Pferdeheilkunde 18, 284-288

Plewa D. (2005): Formularmäßige Verkürzung der Veriährungsfrist für Schadensersatzansprüche gegen den Tierarzł wegen fehlerhafter Kaufuntersuchung. Pferdeheilkunde 21, 580-581

Vertrag über die Untersuchung eines Pferdes (2006): H. D. Lauk und B. Huskamp (Hrsg.), Pferdeheilkunde Berlin 9. Auflage

Dr. Dietrich Plewa

Ludwig-Erhard-Straße 4

76726 Germersheim

rae.plewa-doppler@t-online.de 